\title{
The Application of Physical Internet in Saudi Arabia: Towards a Logistic Hub in 2030
}

RAouf JAZIRI, PhD

College of Business, University of Jeddah

Jeddah, Makkah, Saudi Arabia

Corresponding author: rmjaziri@uj.edu.sa

Khalifa Korbi, PhD student

University of Sfax, Tunisia

Email: khalifa.korbi@gmail.com

Salah Gontara, PhD student

University of Sousse, Tunisia

Email: gontara.salah@gmail.com

\section{About the authors}

Raouf Jaziri received his Ph.D in Management from University of Brest in France. He is currently an assistant professor in College of Business at University of Jeddah in Saudi Arabia. He holds two bachelor degrees in Sciences and Mathematics and Accounting Management. He holds three master's degrees in Entrepreneurship, International Trade and Universities' Management. Raouf Jaziri has been doing methodological research focusing on entrepreneurship, project management, health management, supply chain management, higher education quality management and open educational resources.

Khalifa Korbi holds an Industrial Engineering Degree from the International Multidisciplinary School in Sousse in Tunisia. Currently, he is the director of the Maghreb Institute of Economics and Technology in Sousse and is pursuing his Ph.D in Management in the Faculty of Economics and Management, University of Sfax. Khalifa Korbi's research primarily focuses on logistical networking trends, such as broad logistic networks' interconnections and implementations as presented in the Physical Internet Initiative.

Salah Gontara received his Master degree in Distributed Computing from University of Sousse, Tunisia. Currently, he is pursuing a Ph.D in Computer Science at the same university. He is also holding a position of Adjunct Professor at the place. He received a B.Sc in Theoretical Physical from the Higher School of Science and Technology of Hammam Sousse, Tunisia and a B.Sc in Information Technology from the Higher Institute of Computer Science and Communication Technologies of Hammam Sousse, Tunisia. Salah Gontara's research focuses on decentralized techniques for fault detection and localization in computer networks. 


\section{Abstract}

As a way to diversify its non-oil economy, Saudi Arabia launched a prospective initiative called Vision 2030 to boost its ability as the most prominent middle eastern logistics hub. Given its strategic geographic position at the crossroads of important international trade routes, among three continents - Asia, Europe and Africa, Saudi Arabia has predominant logistical access to the Arabian Peninsula and East Africa, as well as the Asia-Europe trade route. Thanks to the existing infrastructure and all mechanisms already in place in Saudi Arabia, we firmly believe that the Physical Internet presents the true empowerment to these mechanisms by offering improvements in delivery time, costs and environmental impacts.

Keywords: Physical Internet, Logistic hub, Saudi Arabia, Vision 2030

\section{Introduction}

The economic program, Vision 2030, launched by the Saudi government in April 2016, aims to lift the country out of its historical dependence on oil and diversify its economy. This program is based in particular on a transition from the growth relay to the private sector and non-oil activities (Moser et al., 2015). Crown Prince Mohammad Bin Salman drives Saudi Arabia's Vision for the future, which is an ambitious blueprint expressing the long-term goals of the country. The plan, Saudi Vision 2030, is structured around 12 operational programs (Moshashai et al., 2018)

The 8th program, called the National Industrial Development and Logistics Program, is the one focused on logistics, which could benefit the most from the essential characteristics of the Physical Internet. Its objective is to develop the industrial potential of Saudi Arabia by encouraging the localization of manufacturing production in the country. Thus, the Kingdom would eventually become a major logistics hub between three continents. The development of industrial zones goes through the MODON (Saudi Industrial Property Authority), which currently manages 34 industrial cities and plans the development of 5 additional cities over the next five years.

In addition, Saudi Arabia is applying several mechanisms that proved to be successful in making a better logistics network, notably in customs paperwork, making any customs operation faster than ever before. For example, the Saudi government has established four new economic cities, the largest of which is the King Abdullah Economic City (KAEC). It includes the largest port in the Red Sea region (Moser et al., 2015). To accompany this development plan and to be able to face the major challenges of globalization, the State is obliged to remain attentive to innovations. The Physical Internet in Saudi Arabia presented a revolution towards an efficient and sustainable logistics, which aims to significantly improve the economic, environmental and social efficiency and sustainability of the current organization (Alshuwaikhat et al., 2017).

This article attempts to bring the elements of the Physical Internet to the reality of the Saudi logistics sector. It highlights the concept and the research projects carried out on the subject, and looks at an overall presentation as well as a SWOT analysis of the logistics and transport sector in Saudi Arabia. This study 
aims to present the prospects for developing a Physical Internet Network in the Saudi context.

\section{The Physical Internet}

The evolution of current logistical performance must meet contradictory expectations. On the one hand, it has to respect the just-in-time philosophy favored by small and high-frequency shipments. On the other hand, there is the strong desire to improve environmental performance by reducing greenhouse gas emissions. These goals cannot be achieved without limiting the fragmentation of freight flows. In this sense, increasing collaboration among different supply chains or networks is a way to exploit synergies and jointly improve their logistics performance, including transport activities. However, even though horizontal collaboration and bundling of merchandise flows are well-known concepts, only a few implementations are operational today and there is no evidence of widespread scaling up (Sarraj et al., 2013).

We believe that the definition proposed by (Ballot et al., 2010) is exhaustive: "The physical internet is a global logistic system taking advantage of the interconnection of supply networks by a standardized set of collaboration protocols, modular containers and intelligent interfaces for increased efficiency and sustainability". The physical internet, which mimics the digital Internet's model, sets itself to follow the Open Systems Interconnection model (OSI). It falls under the OSI model, offering a more vibrant and rigorous presentation through its seven layers. For this reason, the Open Logistics Interconnection (OLI) Model is established for the Physical Internet. The purpose of this model is to specify a general framework for the creation of subsequent consistent standards. The model itself does not define a service, let alone a protocol.

Fig. 1 shows how these layers interact vertically and how the flow evolves from one layer to the others.

\section{Benchmark Case Studies}

\section{The MODULUSHCA Project in the EU}

Funded by the EU, the MODULUSHCA project (Modular Logistics Units in Shared Co-modal Networks) has not only explored the different methodological and functional components of a physical internet, but also developed and tested its first applications. By organizing different workshops with the experts from their industrial partners, the project members were able to finalize and develop a framework describing how the physical internet enabled the emergence of an interconnected logistics system for everyday consumer goods (FMCG, for Fast-Moving Consumer Goods). They also highlighted the potential barriers and successful parameters of such a system (Sallez et al., 2016).

The MODULUSHCA team has developed modular units for the consumer goods sector. One unit deals more specifically with the locking mechanism, and the other consists of panels. The team also selected and described the digital interconnectivity algorithms among different computer systems, a sensor-sensing approach for the communication of modular logistics units.

The researchers have developed a road map for this interconnected logistics of consumer goods, highlighting the next steps that will lead to the final implementation of this strategy in Europe around 2030 and its 


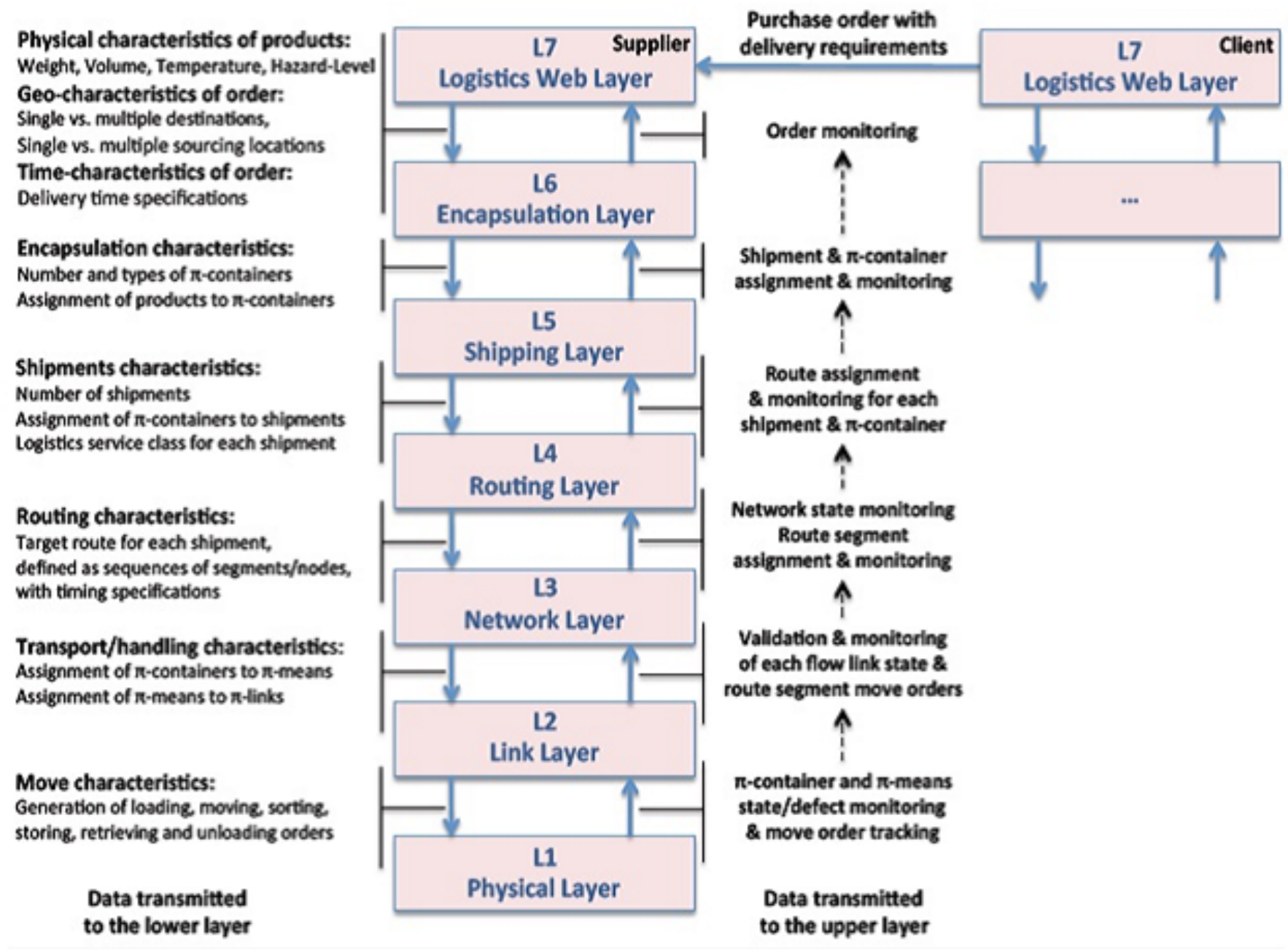

Fig. 1 Inter-layer Service Description of OLI Model (Source: Montreuil, et al., 2012)

deployment to the entire industrial sector around 2050. The roadmap has been structured around the four essential interconnectivity dimensions of the physical internet: physical, digital, operational and commercial (Pan et al., 2017). An advisory committee composed of experts from 13 industrial and scientific institutions was initiated during the project. The MODULUSHCA project partners thus proposed a long-term vision of fully interconnected logistics networks, based on the progress of physical, digital, operational and commercial interconnectivity.

\section{Mass Distribution in France}

The mobility web in the French context is set to facilitate mass distribution through interconnecting several logistic networks, disserved by different third-party logistics (3PLs). As estimated by Ballot et al. (2010), the current logistic networks in France generate waste by the proliferation of untimely transport between production and storage sites, often dedicating to each organization and creating round trips and possible transformations into obsolescence and product waste. The reliance on service providers, which could improve the functioning of logistics, is a partial solution because of the difficulty for an atomized market to find 


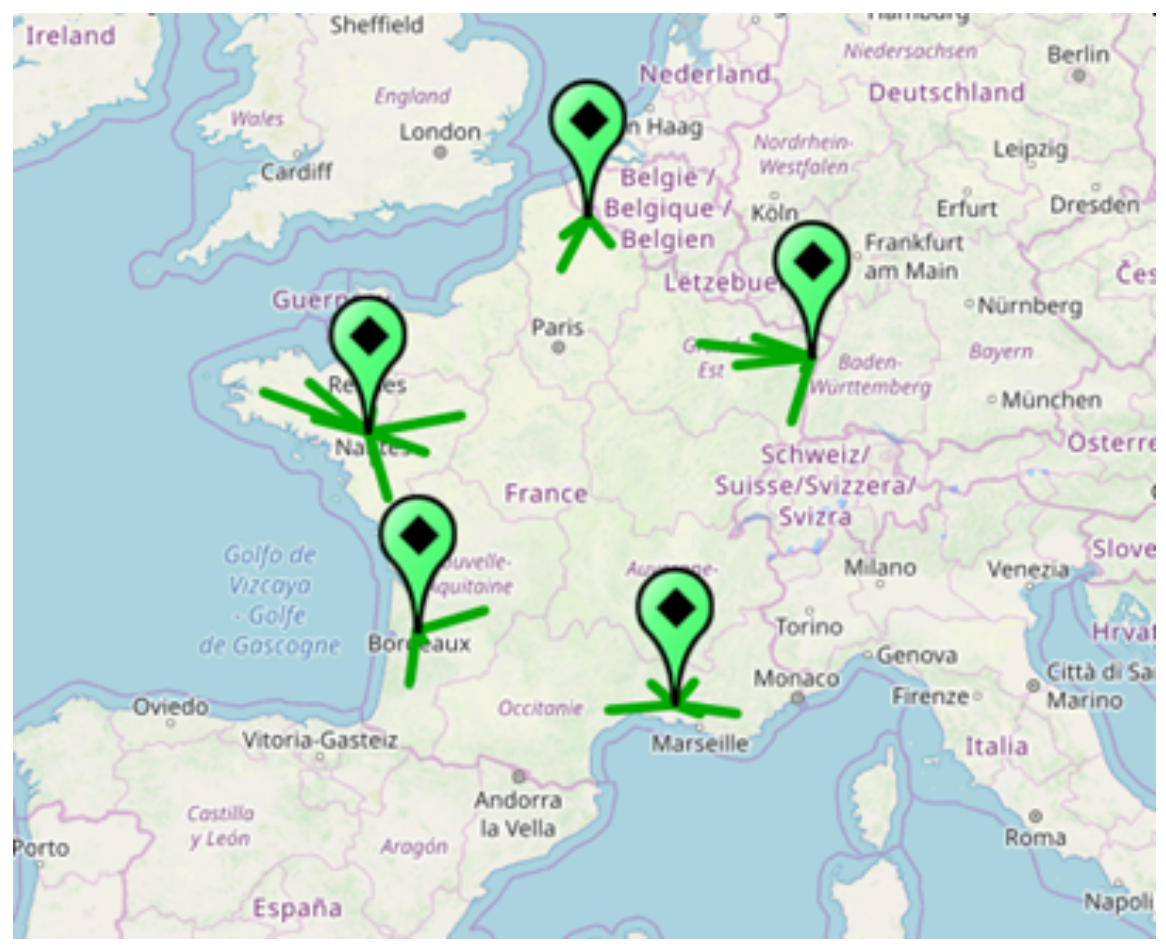

Fig. 2 CRC's Physical Internet Logistics Network in France

Source: http://www.crc-services.com/reseau-crc/(accessedonMarch12, 2018)

synergies between customers with multiple and antagonistic requirements. The need for a new architecture is conspicuous, one with higher sustainability and optimized flow. As a result, The Physical Internet is created as the new architecture to fulfill the need for logistic network interconnection. The Physical Internet has been experimented since January 2015 in France (Ballot, 2019). The start-up CRC (Center for Collaborative Routing) opened five centers near Strasbourg, Lille, Nantes, Bordeaux and Saint-Martin de Crau (Bouchesdu-Rhone). The CRC's primary asset is to open to businesses of all sizes for the possibility of pooling their flows. It presents limited but steady progress in their logistics network, as shown in figure 2.

Eric Ballot claims that after one-year experimentation, the following results were obtained (Ballot, 2019):

- $10 \%$ reduction in $\mathrm{CO} 2$ emissions

- record on-time delivery rate $(99 \%)$

- truck filling rate of up to $90 \%$

- average and target savings of around $30 \%$ to $50 \%$ on transport and storage

\section{Global Synchronization of Logistic Networks between the USA and Canada}

A study on routing optimization is carried out by Fazili et al. (2017) for the eastern Canadian region, comparing the efficiency of the conventional logistics systems, the Physical Internet, the hybrid model, and combinations of these systems. The cost performance of each of these logistic systems varies under various traffic and load selection scenarios. In the network featured by a low ratio of packing to the driving costs, the 


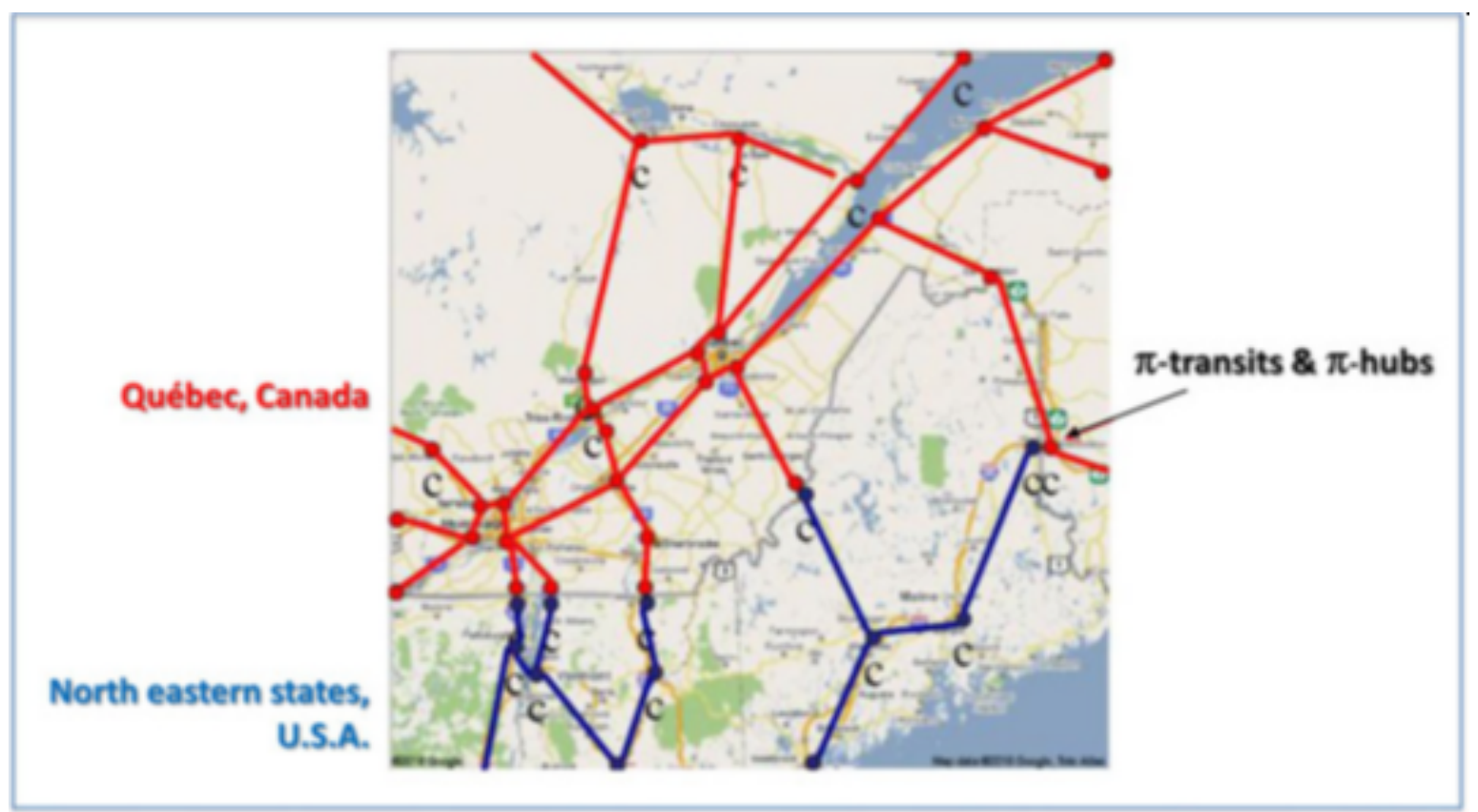

Fig. 3 Physical Internet's Intercity Network at South of Quebec, Canada and North-eastern Area of USA (Source: Montreuil, 2011)

Physical Internet seems to be the superior logistics system followed by a hybrid system and the conventional system (Fazili et al., 2017). In addition, from an environmental point of view, the Physical Internet achieves a substantial reduction in the carbon footprint of the logistics system from driving and reduces truck traffic on the roads (Fazili et al., 2017).

Besides, the Physical Internet allows for the structuring of inter-city trips with PI-transits and PIHubs, which are strategically deployed by various service providers at key locations in the proximity to national borders, ports, airports, highway intersections and other major roads of the city. Figure 3 shows the Canadian links, with pi-nodes in red and the American nodes in blue. It presents double nodes at the borders, permitting safe passage between countries. Cargos are conveyed and vehicles do not need to cross borders (Montreuil, 2011).

Eric Ballot claims that after one-year experimentation, the following results were obtained (Ballot, 2019):

- $10 \%$ reduction in $\mathrm{CO} 2$ emissions

- record on-time delivery rate $(99 \%)$

- truck filling rate of up to $90 \%$

- average and target savings of around $30 \%$ to $50 \%$ on transport and storage 


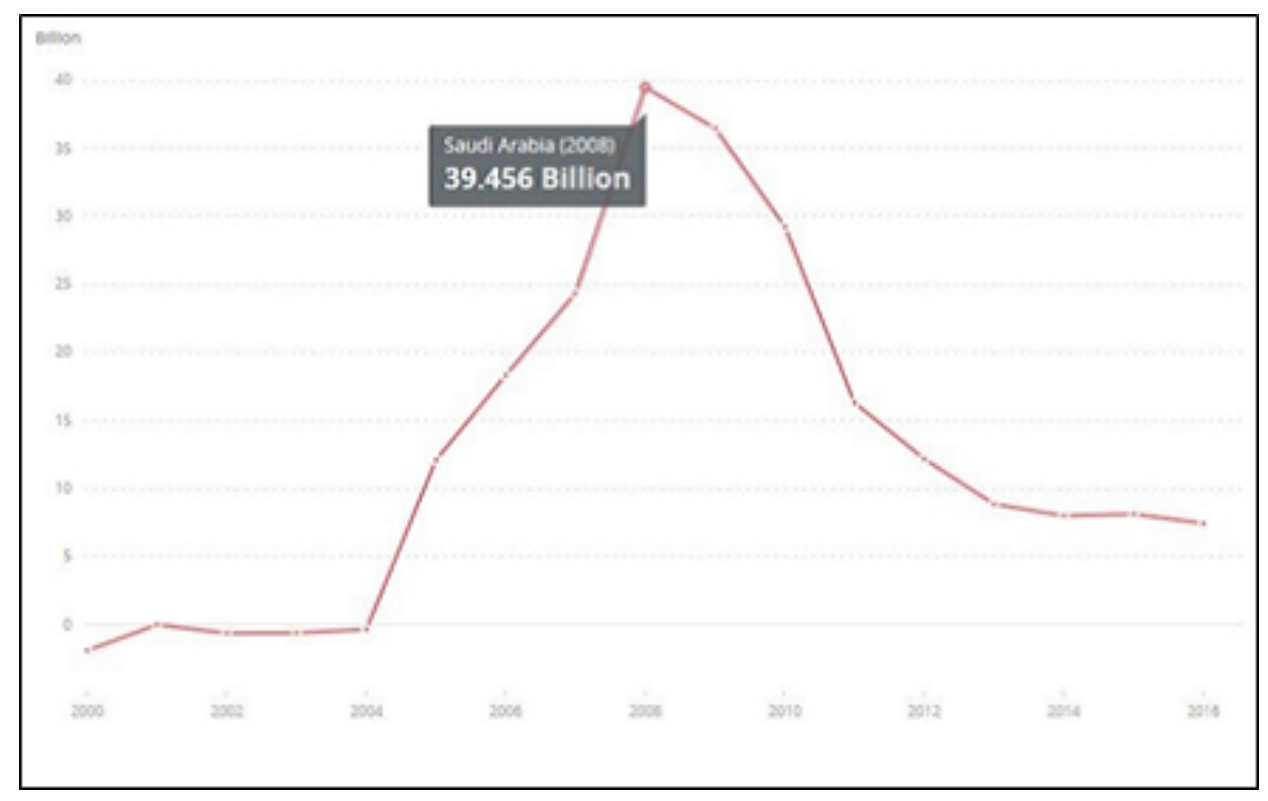

Fig. 4 Foreign Investments in Saudi Arabia

(Source: World Bank. https://data.worldbank.org/,accessedonNov.20,2018)

\section{Current Logistics and Economics in Saudi Arabia}

\section{Economic Situation in Saudi Arabia}

Saudi Arabia is the largest economy in the Middle East and the Arabian countries, and is the richest in the region. The government has undertaken a policy intervention of large-scale public works, increased foreign direct investment and enhanced the soundness of the financial and banking system. These have provided more potential for its economy to stand out in the region. However, Saudi Arabia's economy relies almost entirely on oil. GDP growth has gradually declined since 2015. In 2017, it came to a point where the economy has shown a historic recession $(-0.7 \%)$, while the growth was expected at $1.9 \%$ with the non-oil economy growing further to 2.3\%. The IMF (International Monetary Fund) dropped from $2.4 \%$ to $1.9 \%$ in Saudi Arabia in 2019, as a result of the regional and global geopolitical tensions and the moderation of oil prices since the end of 2018 (IMF).

To become a logistic hub, Saudi Arabia needs to be an investment hub first, and then turn its economy around towards the openness to foreign investments with more focus on its non-oil economy. On the macroeconomic front, Saudi Arabia has been facing a double deficit situation since 2015. The international rating agencies downgraded Saudi Arabia's sovereign rating (Fitch: A+, Moody's: A1), but put it on an outlook list due to its stable fiscal position, high levels of liquidity and large reserves of extractable oils at low cost. On the banking and financial front, in 2015-2016, Saudi Arabia experienced an episode of liquidity crisis caused by the drop in its oil revenue, the freezing of government spending, significant payment arrears and debt issuance on the market. These measures taken by the authorities to resolve the current situation were expected to end the crisis. Nevertheless, the Saudi banking sector has been well regulated and sufficiently 
capitalized to cope with this crisis in case that the profitability of the banks might be heavily impacted.

Thus, as the 7th program of Vision 2030, the Strategic Partnerships Program has been developed to deepen the economic partnerships with the countries considered as strategic and potential actors in the success of the scheme. Among these countries, we consider the following countries as major partners: United States, China, Japan, South Korea, Great Britain, France, Germany and Russia.

At the turn of the new millennium, Saudi Arabia also changed its attitude towards foreign investments which were not taken into account until 2004. The total figure reached a peak of 39.456 billion dollars in 2008. These investments are a major contributor to the development of the transport and logistics sectors. However, they have declined since 2008 as shown in Fig. 4. Saudi Vision 2030 is one of the programs aiming to remediate this trend.

\section{Logistics of Saudi Arabia}

A broad review of literature offers many diverse opinions on logistics outsourcing in different countries. However, little empirical research has been carried out about the situation in the Arab world and Saudi Arabia, specifically. The kingdom of Saudi Arabia is the largest country in the Middle East and the 12th largest in the world, measuring approximately 2.2 million square kilometers, with the largest Gross Domestic Product (GDP) in the Middle East (Sohail et al., 2005). Saudi Arabia has an impressive transportation network made of 27 airports and 12 seaports connected with 65,000 kilometers of roads and 4,130 kilometers of railroads. The data does not include the unpaved roads and the airports with unpaved runways (Ziadah, 2017).

All ports are owned and maintained by private sector companies under long-term contracts with the country. As part of the Saudi export development initiatives, Saudi Arabia grants a 10-day exemption on export charges and 50\% off on port taxes for all export deals. The 27 airports in Saudi Arabia include four in Riyadh, Jeddah, Dammam and Al-Madinah Al-Munawarah. In 2015, more than 81 million passengers transferred in Saudi Arabia, which is a 9.5\% increase compared with 2014. Among them, passengers of air flights reached 646,693. Freight and mail handled reached a total of 1.16 million tons, an increase of $14.1 \%$ over the previous year. Air flight is the preferred transportation method for passenger transferring in Saudi Arabia because of the relatively long distances between major cities. Several major international airlines offer services inside and outside the country (Fig. 5-1, 5-2, 5-3).

\section{Perspectives of the Physical Internet in Saudi Arabia}

\section{Transition to Pl-Hubs}

The PI-Hub, shown in Fig. 4, is an automated cross-dock keeping the same inter-arrival time between incoming and outgoing trucks and the same level of incoming PI-Containers and requests. The characteristics used to describe the cross-dock facility are also the same as the first model (the surface, the distance and the number of incoming and outgoing docks). However, instead of using forklifts, automated loading and 


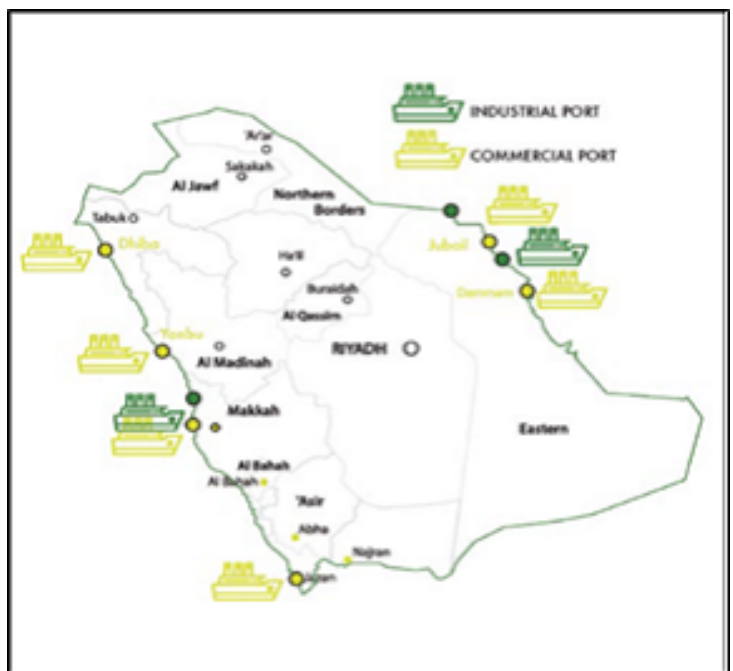

Fig. 5-1 Maps of Road Network in Saudi Arabia

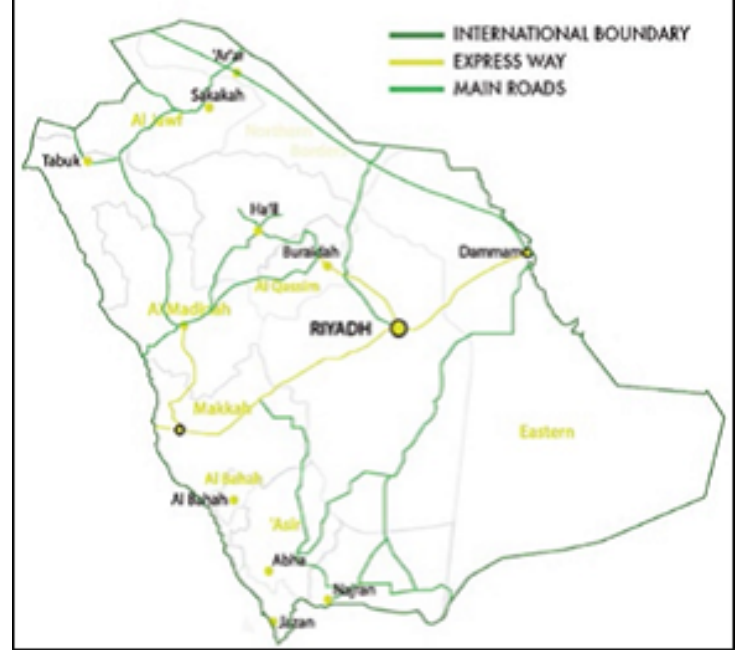

Fig. 5-2 Maps of Railways in Saudi Arabia

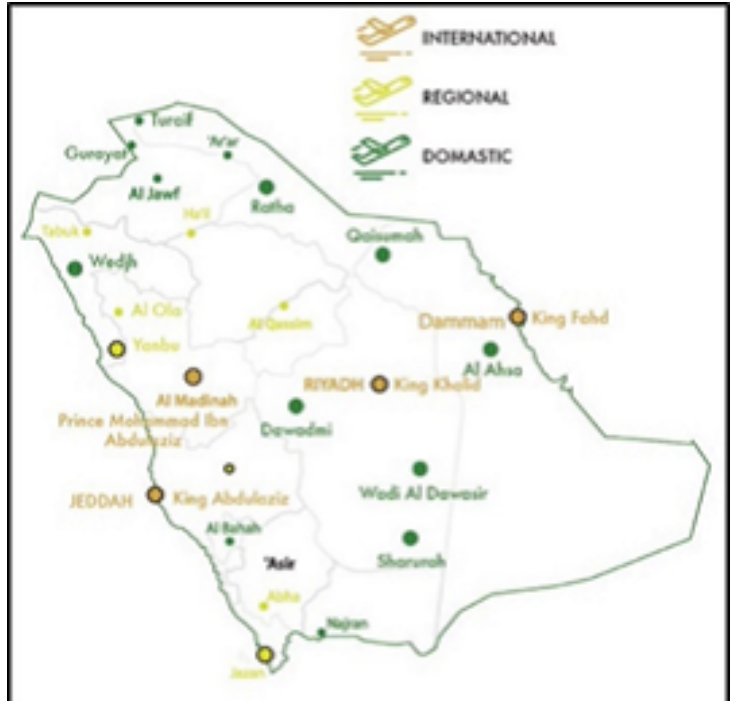

Fig. 5-3 Maps of Railways in Saudi Arabia

(Source: https://www.ic.gov.sa/en/,accessedonSept.8,2018) 

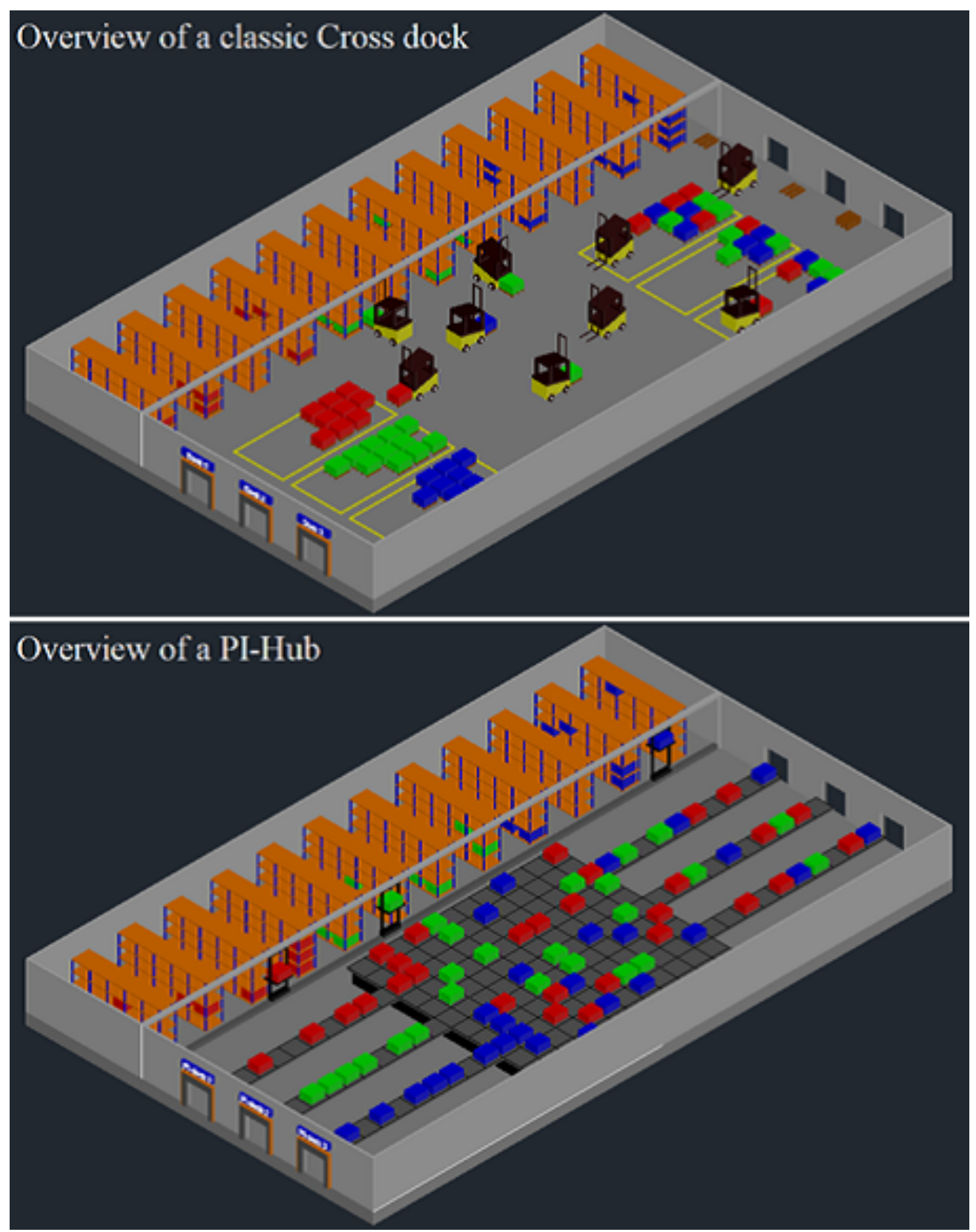

Fig. 6 Classic Cross-docks vs PI-Hubs (Source: Chargui et al., 2016)

unloading resources (PI-docks) connected to a sorting area (PI-sorters) by PI-conveyors are applied (Chargui et al., 2016).

The average speed adopted for the PI-conveyors is $2.77 \mathrm{~m} / \mathrm{s}(10 \mathrm{~km} / \mathrm{h})$. In the PI-cross dock, manual forklifts for storing and retrieving products in the temporary warehouse are replaced by an Automated Storage and Retrieval System (AS/RS), which is connected directly to the sorting area with three storage and retrieval machines. When a truck arrives at an incoming dock, the PI-dock unloads the cargos automatically and the cargos will be moved to the PI-sorters by PI-conveyors. The trucks' orders are served in the PI-Hub. The required PI-Containers are picked up by the AS/RS system. Then they come through the sorting area (PI-sorters) to the PI-dock through PI-conveyors. If there is no sufficient quantity to serve the trucks' orders, 
or if there is a truck being unloaded in an incoming PI-dock, then the PI-Containers go directly from that incoming PI-dock to the outgoing PI-dock where the PI-Container is requested (Chargui et al., 2016).

\section{Transition to Pl-Containers}

The PI-Containers are easy to handle, store, transport, seal, snap to a structure, interlock together, load, unload, build and dismantle (Montreuil et al., 2015). The PI-Containers are efficiency for the following characteristics (Montreuil et al., 2010):

- Coming in various modular sizes, from cargo container sizes down to tiny sizes.

- Easy to handle, store, transport, seal, clench, interlock, load, unload, construct, dismantle, panel, compose and decompose.

- Made of environment-friendly materials, with minimal off-service footprint.

- Minimizing packaging materials requirements through the enabling of fixture-based protection and stabilization of their embedded products.

- Coming in various usage-adapted structural grades.

- Having conditioning capabilities (e.g., temperature) as necessary.

- Sealable for security purposes.

The need for Saudi Arabia to switch to PI-Containers is to adhere to the Physical Internet initiative from a practical point of view. The switch would allow goods to be shipped and transited through Saudi Arabia in conformity with the new EU standards in handling FMCG and as mentioned in the MODULUSHCA project (Sallez et al., 2016).

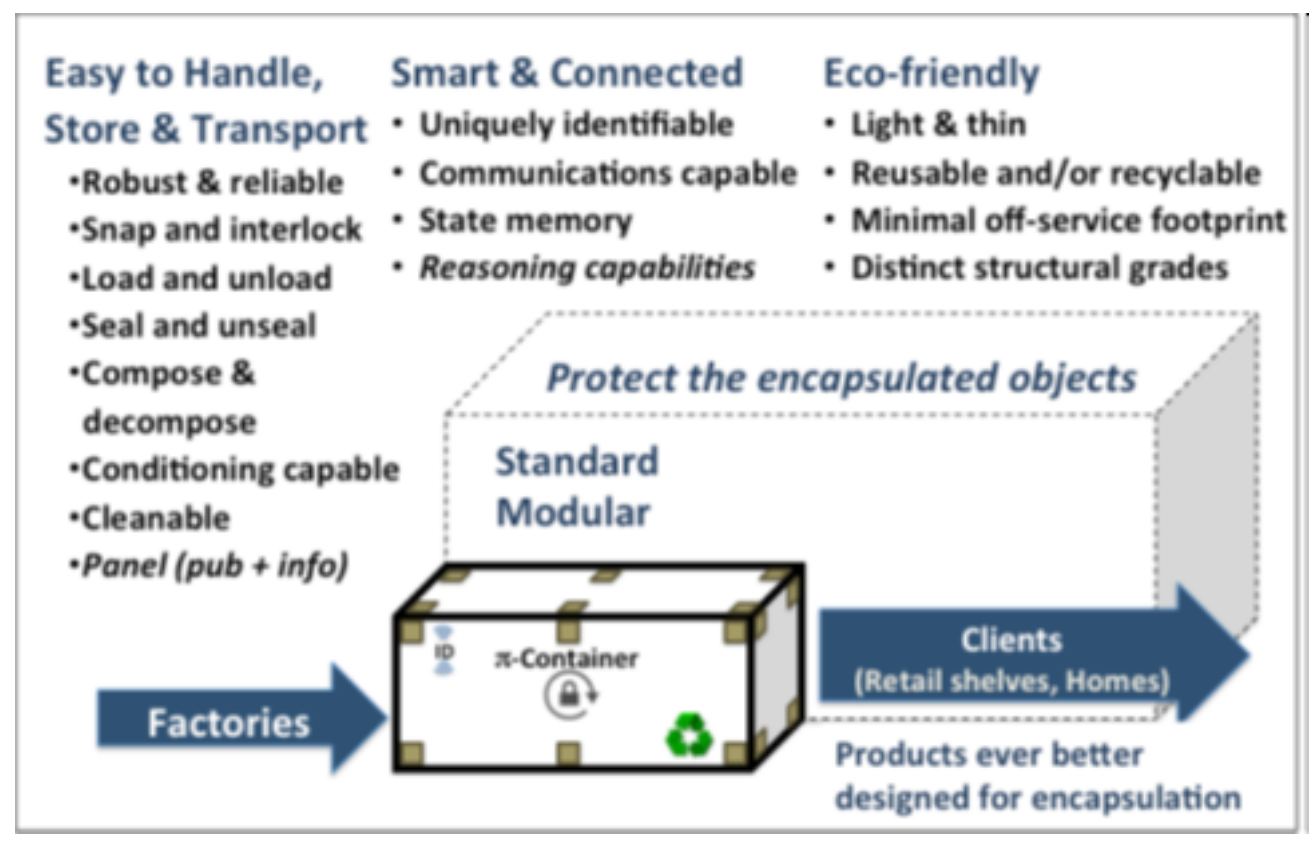

Fig. 7 Generic Characteristics of Physical Internet Containers

(Source: Montreuil et al., 2015) 


\section{SWOT Analysis}

The SWOT analysis of the main characteristics of the internal and external environments of the Saudi logistics sector, as shown in Table 1, makes it possible to identify multiple assets that have not been sufficiently exploited so far. The result indicates the logistics sector still suffers from several deficiencies that affect it at different levels: economic, technological, environmental, legislative and social. These problems find their way into the foundations of the Physical Internet.

Indeed, the Physical Internet envisages universal interconnectivity that will be concretized by a set of collaboration protocols, allowing all Saudi logistic actors to adjust their service to universal standards. This will have important repercussions on several elements: the improvement of the service quality, the human skills and the working conditions; reinforcement of the maturity of the principals and the transparency on the market; as well as the consolidation of the laws governing the sector. The standardization of the collaboration

Table 1 SWOT Analysis for Saudi Logistics

\begin{tabular}{|c|c|}
\hline Strengths & Weaknesses \\
\hline $\begin{array}{l}\text { - A quarter of the world's oil reserves and } \\
\text { OPEC's largest producer } \\
\text { - Major economic and regional role } \\
\text { - Economy in the process of diversifica- } \\
\text { tion strengthened by the Saudi Vision } \\
2030 \text { program } \\
\text { - Solid financial position due to low debt } \\
\text { and considerable assets } \\
\text { - Robustness of the banking system }\end{array}$ & $\begin{array}{l}\text { - High dependence on the hydrocarbon } \\
\text { sector, low job creation, and increasing } \\
\text { domestic demand for energy } \\
\text { - High unemployment rate of nationals } \\
\text { - Fragility of governance darkening the } \\
\text { business climate } \\
\text { - Unstable geopolitical environment } \\
\text { Economy dependent on public expendi- } \\
\text { ture }\end{array}$ \\
\hline Opportunities & Threats \\
\hline $\begin{array}{l}\text { - Saudi Arabia benefits from a strategic } \\
\text { geographical position, within a conjunc- } \\
\text { ture point of three continents: Asia, Eu- } \\
\text { rope and Africa } \\
\text { - The creation of a Public Investment } \\
\text { Fund: the new domestic assets under } \\
\text { management of the Public Investment } \\
\text { Fund are expected to reach } 20 \% \text { of the } \\
\text { total portfolio by } 2020 \\
\text { - Implementation of a privatization pro- } \\
\text { gram: BOO (Build, Operate, Own,) } \\
\text { and BOT (Build, Operate, Transfer) } \\
\text { models will be generalized for infras- } \\
\text { tructure construction. }\end{array}$ & $\begin{array}{l}\text { - Legislation protecting the interests } \\
\text { of principals is distinguished by its } \\
\text { fragility } \\
\text { - Access to land is one of the main obsta- } \\
\text { cles in the sector } \\
\text { - The national economy is characterized } \\
\text { by vulnerability to EU, asian and north } \\
\text { american countries. } \\
\text { - Competition from public logistics struc- } \\
\text { tures is tough } \\
\text { - Human and financial investments in re- } \\
\text { search and development are very lim- } \\
\text { ited }\end{array}$ \\
\hline
\end{tabular}


protocols will also facilitate effective circulation of physical and moral entities to avoid ravaging the world.

The Physical Internet offers an efficient and resilient open global system that realizes a reliable distribution of encapsulated goods to a multitude of open Pi-Hubs around the world. This system adopts multimodal transfer, removes the detours at warehouses and lowers the transport costs and storage costs. Thus, a significant reduction in logistics costs is expected for the Saudi business market. In all, the homogeneity of intelligent technologies and the application of automation structure on a global scale, proposed by the Physical Internet, presage an optimization of the current logistical costs.

\section{Simulation and Results}

We simulate PI-Containers road-deliveries in a Physical Internet environment between several PI-Hubs, which are situated in major cities and industrial compounds, and next to seaports and airports as a hypothesis. Generally speaking, road delivery system is the easiest to tweak yet they are the least optimized. We run our simulation on AnyLogic with the OpenStreetMap API following the PI-BGP routing algorithm (Gontara et al., 2018) and the PI-enabled mobility web (Hakimi et al., 2012).

We use the following estimates as parameters in our simulation (Wiki, 2015)

- Delivery/pick-up per person per day;

- Delivery/pick-up per economic activity per week;

- Between 300 and 400 freight vehicle trips per 1,000 persons per day;

- Between 30 and 50 tonnes per person per year.

Fig. 8 shows the simulation result of the Physical Internet network in Saudi Arabia in AnyLogic with map data from OpenStreetMap. The simulation runs the equivalent of 1000 days of deliveries through the Physical Internet. The generated data in Fig. 9 shows the time it takes to fulfill each kind of delivery for all the possible PI-Hubs combinations as indicated on the map. The mean delivery time is 21 hours, and the maximum delivery time is 47 hours for the furthest PI-Hubs, which is under the two days mark. It is significant progress considering how large the kingdom of Saudi Arabia is.

\section{Conclusion}

In order to gain from the benefits of resource sharing, the Physical Internet must be adopted on a universal scale. Hence, the idea of studying development prospects in an emerging country such as Saudi Arabia will contribute to the global growth of the world in the related fields. The study of the internal and external environments of the Saudi logistics sector has shown that the Physical Internet will be a continuity in ambitions described in the national strategy of Saudi Arabia to enhance its logistics competitiveness.

Our single simulation reported above demonstrates the benefits of using the Physical Internet in Saudi Arabia for significan gains in delivery times through adoptiono of new routing protocols and shipping techniques. Other benefits, ranging from social to environmental levels, are also at hand and need to be measured 


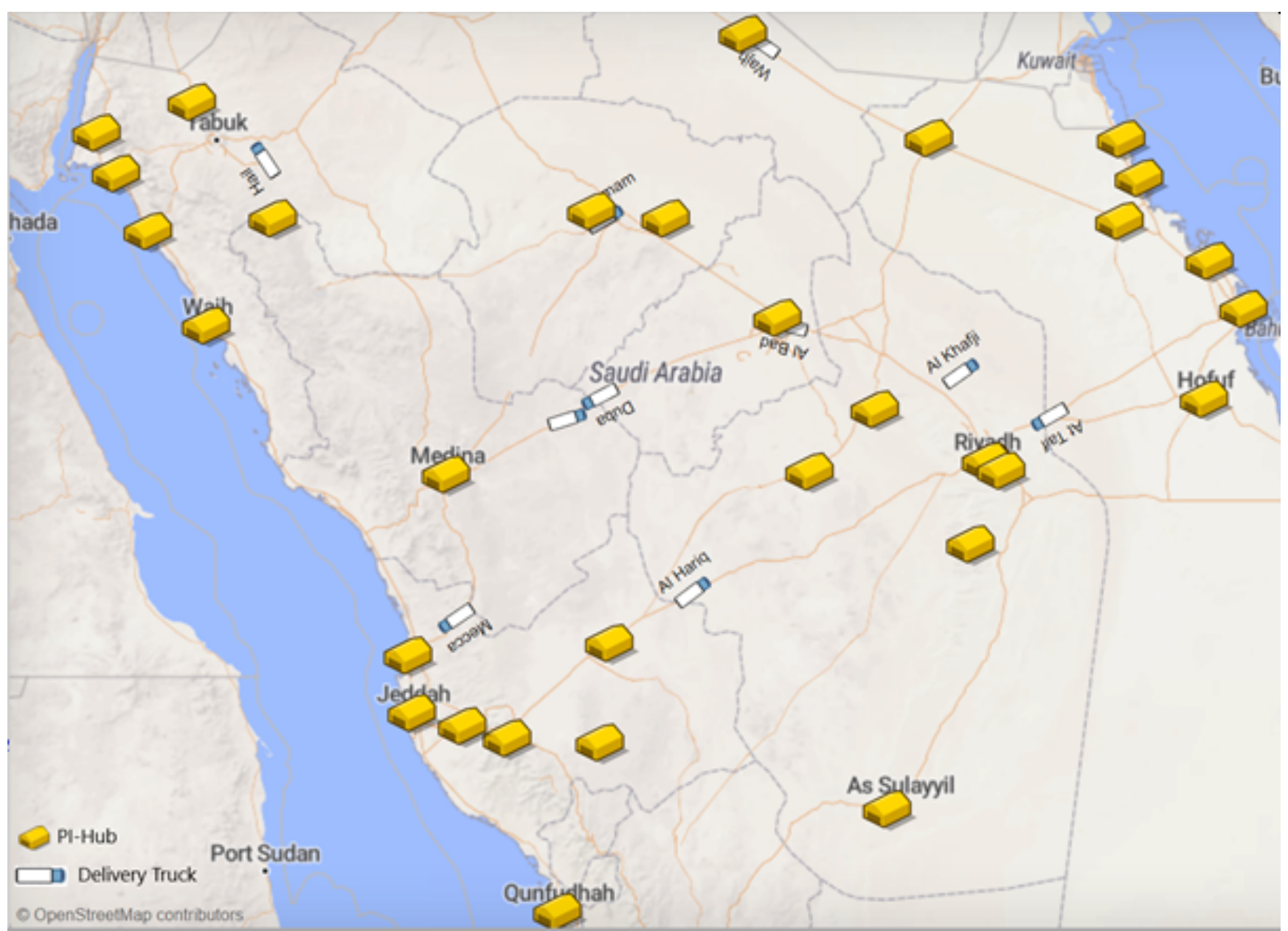

Fig. 8 Simulation of Physical Internet Network in Saudi Arabia via AnyLogic and OSM

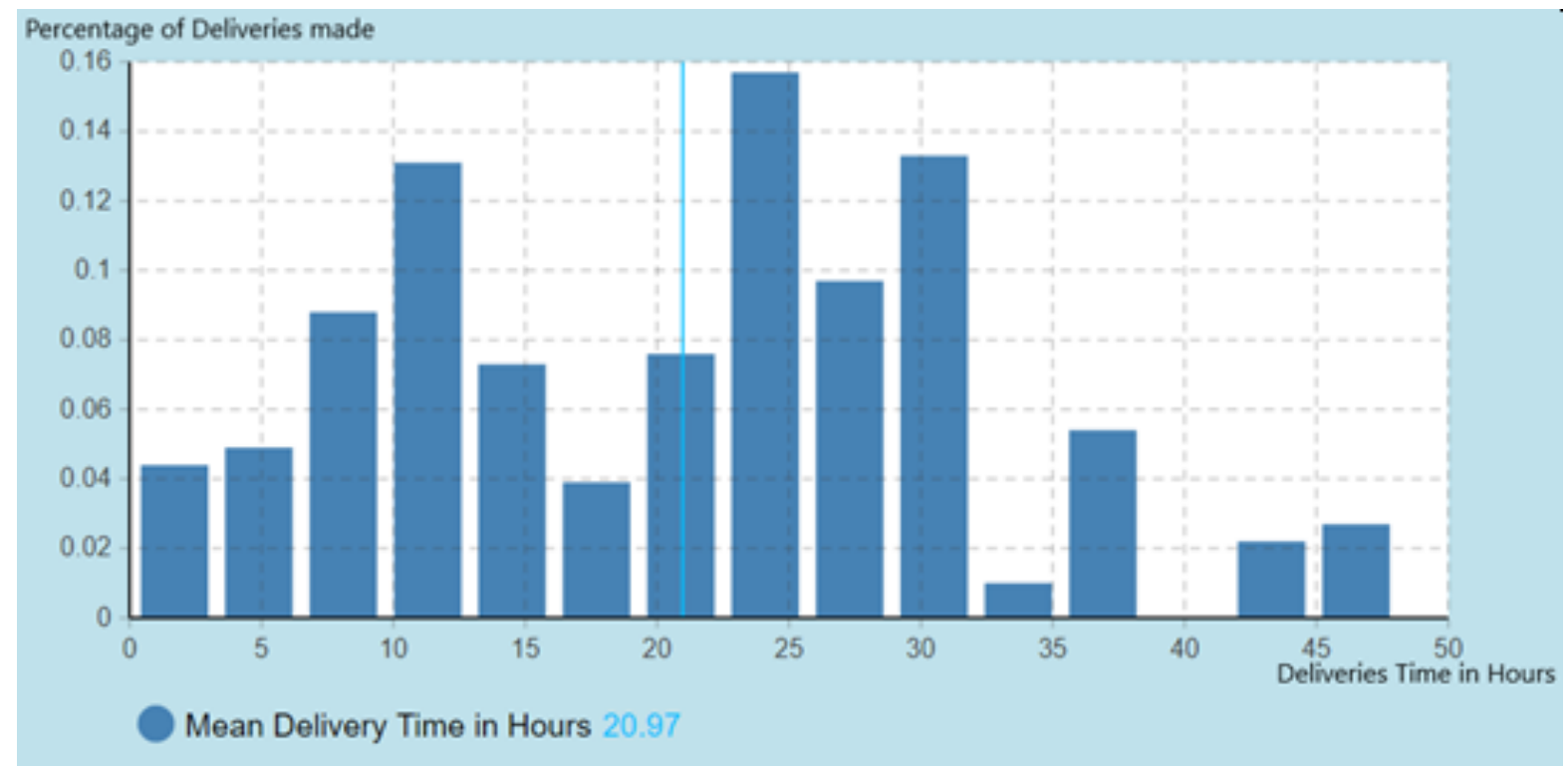

Fig. 9 Deliveries Time Chart 
through further experimentations.

The Saudi Vision 2030 of its 8 th program proposes a constructive idea about the enhancement of its logistics sytem through the creation of the Physical Internet model. Presumably, it will become a competitior of the advanced logistics networks implemented in the European Union and provides the possibility to be integrated into the conceptual framework of the global logistics structure.

\section{References}

Alshuwaikhat, H. M. and Ishak, M., 2017. Sustainability Matters in National Development Visions Evidence from Saudi Arabia's Vision for 2030. Sustainability. Vol. 9. Accessed on Sept. 30,2019 from file://C:/Users/Rong\%20Zhang/AppData/Local/Packages/Microsoft.MicrosoftEdge_8wekyb3d8bbwe/TempState/D 09-00408\%20(1).pdf.

Ballot, E., Glardon, R. and Montreuil, B., 2010. Rapport Open Fret: Contribution à la conceptualisation et à la réalisation d'un Hub Rail_Route de l'Internet Physique. PREDIT: Programme de Recherhe et D'innovation Dans les Trasports Terrestres, 2010.

Ballot, E., 2019. Operations, Logistics and Supply Chain Management, Springer, 2019.

Chargui, T., Reghioui, M., Bekrar, A. and Trentesaux, D., 2016. A Simulation Model to Migrate from Cross Docks to PI-hubs. Paper Presented at 3rd International Physical Internet Conference, Atlanta, USA.

Fazili, M., Venkatadri, U., Cyrus, P. and Tajbakhsh, M., 2017. Physical Internet, Conventional and Hybrid Logistic Systems: A Routing Optimisation-based Comparison Using the Eastern Canada Road Network Case Study. International Journal of Production Research, Vol. 55. pp. 2703-2730.

Gontara S., Boufaied A. and Korbaa, O., 2018. Routing the Pi-Containers in the Physical Internet Using the PI-BGP Protocol. 2018 IEEE/ACS 15th International Conference on Computer Systems and Applications (AICCSA). Aqaba Jordan: IEEE, 2018.

Hakimi, D., Montreuil, B., Sarraj, R., Ballot, E. and Pan, S., 2012. Simulating a Physical Internet Enabled Mobility Web: The Case of Mass Distribution in France, MOSIM ' 12 held on June 06-08, Bordeaux, France.

IMF International Monetary Fund [Online ]ref8 Accessed on Sept. 30, 2019 from https://www.imf.org/ en/Countries/SAU.

Montreuil, B., Ballot, E. and Fontane, F., 2012. An Open Logistics Interconnection Model for the Physical Internet. 14th IFAC Symposium on Information Control Problems in Manufacturing. Bucharest, Hungary.

Montreuil, B., 2011. Toward a Physical Internet: Meeting the Global Logistics Sustainability Grand Challenge. Logistics Research, Vol. 3. pp. 71-87.

Montreuil, B., Meller, R. D. and Ballot, E., 2010. Towards a Physical Internet: The Impact on Logistics Facilities and Material Handling Systems Design and Innovation. Progress in Material Handling Research, pp. 305-327.

Sarah, M., Marian, S. and Alkhabbaz M. H., 2015. King Abdullah Economic City: Engineering Saudi Arabia's Post-oil Future. Cities, Vol. 45, pp. 71-80. 
Moshashai, D., Leber, A. M. and James D. S., 2018. Saudi Arabia Plans for Its Economic Future: Vision 2030, the National Transformation Plan and Saudi Fiscal Reform. British Journal of Middle Eastern Studies, pp. 1-28.

Pan, S., Ballot, E., Huang, GQ. and Montreuil, B., 2017. Physical Internet and Interconnected Logistics Services: Research and Applications. International Journal of Production Research, Vol. 55, pp. 26032609.

Salleza, Y., Pan, S., Montreuil, B., Berger, T. and Ballot, E., 2016. On the Activeness of Intelligent Physical Internet Containers. Computers in Industry, Vol. 81, pp. 96-104.

Sohail, S., Al-Abdali M. S. and Al-Abdali O. S., 2005. The Usage of Third Party Logistics in Saudi Arabia: Current Position and Future Prospects. International Journal of Physical Distribution 83 Logistics Management, Vol. 35 No. 9, pp. 637-653.

Wiki, C., 2015. Smart Choices for Cities. Making Urban Freight Logistics More Sustainable. Available on May 20, 2019 at http://www.eltis.org/sites/eltis/files/trainingmaterials/civ_pol-an5_ urban_web-1.pdt.

Ziadah, R., 2017. Constructing a Logistics Space: Perspectives from the Gulf Cooperation Council. Environment and Planning D: Society and Space, Vol. 36, pp. 666-682. 